ORIGINAI

\title{
BROTE DE CRIPTOSPORIDIOSIS EN GUADARRAMA (COMUNIDAD AUTÓNOMA DE MADRID)
}

\author{
Enrique Rodríguez-Salinas Pérez (1), Andrés-José Aragón Peña (2) Marta Allue Tango (2), María \\ Angeles Lópaz Pérez (2), Mariana Jiménez Maldonado (2) y María José Domínguez Rodríguez (2)
}

(1) Centro de Salud de Guadarrama. Madrid

(2) Servicio de Salud Pública del Area 6. Comunidad de Madrid

\section{RESUMEN}

Fundamento: En la primavera de 1998 hubo un brote de gastroenteritis aguda en Guadarrama (Comunidad de Madrid, España) que afectó sobre todo a niños en edad preescolar.

Métodos: Se hizo un estudio descriptivo y analítico (casos y controles) del brote. Se tomaron muestras para estudio microbiológico de las heces de los afectados. Se inspeccionó el sistema de captación de aguia de consumo de propiedad municipal y se tomaron muestras para análisis microbiológico.

Resultados: Veintiún niños/as de 0 a 5 años presentaron diarrea líquida de más de 5 días de duración. Se encontró una asociación estadísticamente significativa entre el consumo de agua del grifo y la gastroenteritis $[O R=5,73$ $(1,18-43,30) ; p<0,05]$. No se halló asociación con otras variables investigadas. Se detectaron ooquistes de Cryptosporidium parnum en heces de ocho de las personas afectadas. Se observaron deficiencias en el sistema de captación y depuración de agua de consumo público de propiedad municipal pero no se encontraron ooquistes en los anúlisis del agua.

Conclusiones: Los resultados de la investigación epidemiológica sustentan que el origen del brote de gastroenteritis por Cryptosporidium parvun fue el agua del grifo. Aunque no se detectó este parásito en los análisis de agua, esto es explicable por las dificultades que entraña esta técnica. En la revisión bibliográfica, no se han hallado descripciones de brotes epidémicos similares en nuestro país, pero según algunos informes, no son infrecuentes en países de nuestro entorno. Por tanto, Cryptosporidium parvim debería ser tenido en cuenta en el diagnóstico y manejo de estas situaciones.

Palabras clave: Criptosporidiosis. Brote epidémico. Parasitosis. Aguas de consumo. Gastroenteritis.

Correspondencia:

Enrique Rodríguez-Salinas Pérez

Centro de Salud de Guadarrama

Av. Reyes Católicos, 2

Guadarrama

28440 Madrid

Correo electrónico: med013434@nacom.es

\section{ABSTRACT}

\section{Outbreak of Cryptosparidisis in Guadarrama (Autonoumous Community of Madrid)}

Background: In spring of 1998, an acute gastroenteritis outbreak, which mainly affected preschool children, took place in Guadarrama (Community of Madrid, Spain).

Methods: A descriptive and analytical (cases and controls) study of the outbreak was done. Stool samples of the cases were taken to microbyological investigation. The tap water getting system of the municipality was inspected and samples were collected to microbyological analysis.

Results: Twenty one children from 0 to 5 years-old had liquid diarrhea which lasted more than five days. A significant statistical association was found within tap water consumption and gastroenteritis $[O R=5,73(1,18-43,30)$; $p<0,05]$. It did not have association with other variables investigated. Cryptosporidiam parium $(C P)$ oocysts were detected in stool samples of eight cases. Deficiencies were observed in the tap water getting and treatment system of the municipality but no oocysts were found in the analysis of the water:

Conclusions: The results of the epidemiological investigation support that the source of the gastroenteritis outbreak was tap water, likely contaminated with $C p$. While this parasite was not detected in the analysis of the water, it is explainable for the difficulties which this technique involves. In our review similar descriptions of outbreaks have not been found in Spain, but it seems that this reports are not infrecuent in neighbour countries. Therefore, $C p$ should be borne in mind in diagnostic and management of these situations.

Key words: Cryptosporidiosis, Cryptosporidium Purvum, Outbreaks, Water, Gastroenteritis, Diarrhea.

\section{INTRODUCCIÓN}

Desde la descripción del primer caso de criptosporidiosis humana por Nime et al. ${ }^{1}$ en 1976 , se ha producido un gradual incremento 
de publicaciones sobre el tema, paralelo a su creciente importancia como "enfermedad emergente"2.

La sucesiva comunicación, tanto de casos aislados en pacientes inmunodeficientes, graves y de tórpida evolución ${ }^{3-6}$, como de brotes de gastroenteritis severa aunque autolimitada en población general ${ }^{7-1} 8$, ha ido abriendo el campo de conocimiento sobre la infección en humanos por Cryptosporidium parvum $(C p)$.

La enfermedad se produce tras la ingestión de ooquistes ${ }^{19}$. Estos son eliminados masivamente al medio ambiente con las deposiciones de animales y seres humanos infectados ${ }^{20-22}$. Se ha descrito el contagio a través de mecanismos de transmisión diversos: persona-persona ${ }^{10,16,23}$, animal-perso$\mathrm{na}^{16}$, aguas de bebida o recreativas ${ }^{9,11-14,17,24}$, alimentos ${ }^{7}$, etc.

La gran magnitud de algunas epidemias ${ }^{25}$, la afectación preferente en la infancia y en personas malnutridas e inmunodeficientes, así como la capacidad de este protozoo para evadir los controles habituales del agua de bebida y su resistencia a la cloración, deben alertar sobre su potencial importancia como problema de salud en la comunidad 12.17.26-29.

Algunas epidemias han producido una gran polémica social y han llevado a la elaboración de informes de expertos, como el de Badenoch en el Reino Unido ${ }^{4,11.12}$, con recomendaciones para la mejora de la calidad de las aguas de bebida y sistematización de la búsqueda en las muestras de laboratorio clínico.

En el presente trabajo se estudia un brote epidémico de gastroenteritis por $C p$, ocurrido en la primavera de 1998 en el municipio de Guadarrama, en la Comunidad de Madrid. El aumento de casos de gastroenteritis aguda en la consulta de pediatría del Centro de Salud municipal y la observación por el director de un colegio de un gran número de ausencias a clase, sobre todo de los escolares de menor edad, les condujo en ambos casos a comunicarlo al Servicio de Salud Pública del Área 6 de la Comunidad de Madrid, desde donde se realizó la investigación del brote.

\section{MATERIAL Y MÉTODOS}

\section{A) Investigación epidemiológica}

\section{Investigación preliminar}

Se revisaron las historias clínicas de todos los niños que acudieron a la consulta desde el 14 de abril de 1998, así como los informes de urgencias. Desde el día 24 , que se declaró el brote, se captó activamente a todos los posibles casos para anamnesis protocolizada. Se indagó también en las consultas de otros médicos, del Centro de Salud como privados.

La información inicial obtenida entre los niños con diarrea permitió saber que éstos acudían a varios centros escolares del municipio. Se hicieron encuestas epidemiológicas en esos centros para investigar una posible fuente alimentaria común, ya que varios de los comedores de estos colegios eran servidos por el mismo catering de comidas. Además se inspeccionaron los comedores de los colegios y la cocina de la empresa de catering. También se tomaron muestras de heces de los enfermos para su análisis microbiológico. Los resultados, tanto de la encuesta epidemiológica como de las inspecciones efectuadas, no fueron concluyentes. Sin embargo, el laboratorio comunicó la detección de $C p$ en las heces de varios de los niños afectados. Se decidió hacer entonces un estudio de casos y controles para conocer la fuente de infección.

\section{Definición de caso}

Se consideró caso probable a toda persona residente en el municipio de Guadarrama, menor de 6 años, afectado por diarrea (tres o más deposiciones por día) de 5 o más días de evolución, que acudió a la consulta del Cen- 
tro de Salud por este motivo en las fechas en que tuvo lugar el brote. Se definió como caso confirmado a toda persona residente en el municipio de Guadarrama, afectado por diarrea, que acudió a la consulta del Centro de Salud por este motivo en las fechas en que tuvo lugar el brote y en el que se visualizó la presencia de ooquistes de $C p$ en heces mediante la técnica modificada de Ziehl-Neelsen ${ }^{30}$.

\section{Cuestionario}

Se utilizó la bibliografía disponible sobre investigación de brotes de diarrea producida por $C p$ para elaborar un cuestionario ${ }^{7-12,21,24}$. La información se obtuvo mediante encuesta telefónica realizada al padre o a la madre de los niños afectados. Las variables incluidas en la encuesta fueron: edad, sexo, domicilio, número de hermanos, escolarización, centro escolar, uso del comedor escolar, enfermedades previas o concomitantes, fecha de inicio de los síntomas y duración, características clínicas de la enfermedad, otras personas con diarrea en el entorno familiar o residencial, tipo de agua consumida (del grifo y/o mineral), consumo de leche no pasteurizada, contacto con animales, excursiones al campo y baño en piscina, pantano, etc.

\section{Estudio de casos y controles}

El estudio de casos y controles se llevó a cabo entre los meses de abril y junio de 1998. La población de estudio está definida por las personas que acudieron a consulta de pediatría del Centro de Salud de Guadarrama entre el 14 de abril y 1 de mayo de 1998 (período comprendido entre el primer y último caso conocido en el momento del estudio).

Los controles se obtuvieron entre los niños de 0 a 6 años que habían acudido al Centro de Salud en las fechas del brote por motivo de consulta sin relación con problemas gastrointestinales. Aunque no se realizó emparejamiento, no se hallaron diferencias estadísticamente significativas en cuanto a edad en el grupo control y en el de los casos. En total se pudo en- cuestar a 24 controles. La encuesta fue igual que la utilizada para los casos.

No se incluyó en el estudio a los niños no residentes en el municipio de Guadarrama, aunque estuvieran escolarizados o acudieran al Centro de Salud de dicho municipio. Para el estudio de casos y controles se incluyeron como casos tanto a los probables como a los confirmados.

\section{Análisis estadístico}

Tanto para el estudio descriptivo de los casos como para el estudio de casos y controles se utilizaron los programas EPIINFO 6 y SPSS. Se obtuvieron las OR crudas y ajustadas para cada factor estudiado junto con la prueba $\chi^{2}$ de Mantel-Haenzel para su significación estadística.

\section{B) Investigación sobre el abastecimiento de agua de consumo público}

El municipio de Guadarrama dispone de dos tipos de sistemas de abastecimiento de agua de consumo público. Por lo general, hasta el momento del brote, durante la primavera y el verano se abastecía de agua gestionada por una empresa pública de ámbito autonómico y durante el otoño e invierno, según el régimen de lluvias, utilizaba el sistema de abastecimiento de propiedad municipal. Es este último el que estaba suministrando el agua a la población en el momento en el que ocurrió el brote. Se inspeccionó la captación y sistema de tratamiento de aguas que corresponden a la traída municipal y se tomaron muestras de agua para su posterior análisis microbiológico.

\section{RESULTADOS}

\section{A) Descripción de los casos}

Veintiún niños cumplieron los criterios de caso, de los cuales $9(43 \%)$ fueron confir- 
mados por el laboratorio. Doce (57\%) eran niñas. La edad media fue de 2,2 años (DE:1,22 años). La distribución por edades de los casos se muestra en la tabla 1. La enfermedad tuvo una duración media de 9,8 días (DE:5,73 días; rango: 4 a 30 días; mediana: 8 días). Veintiún casos $(100 \%)$ tuvieron diarrea, $14(67 \%)$ dolor abdominal, 6 (29\%) fiebre y 4 (19\%) vó- mitos. Catorce niños (67\%) estaban escolarizados en el momento del brote y de éstos 5 (36\%) acudían habitualmente al comedor del centro escolar. La presentación temporal de estos casos se muestra en la figura 1 . En ninguna de estas variables se hallaron diferencias estadísticamente significativas entre casos probables y confirmados.

Tabla 1

Distribución de los casos por edad y sexo

\begin{tabular}{|l|c|c|c|}
\hline \multirow{2}{*}{ Edad } & \multicolumn{2}{|c|}{ Sexo } & \multirow{2}{*}{ Total } \\
\cline { 2 - 4 } & Varones & Mujeres & \\
\cline { 2 - 4 } & 0 & 1 & 1 \\
\hline Menores de 1 año & 2 & 3 & 5 \\
\hline 1 año & 4 & 3 & 7 \\
\hline 2 años & 3 & 3 & 5 \\
\hline 3 años & 0 & 2 & 2 \\
\hline 4 años & 0 & 1 & 1 \\
\hline 5 años & 9 & 12 & 21 \\
\hline Total & & & \\
\hline
\end{tabular}

Figura 1

Distribución temporal del inicio de los casos.

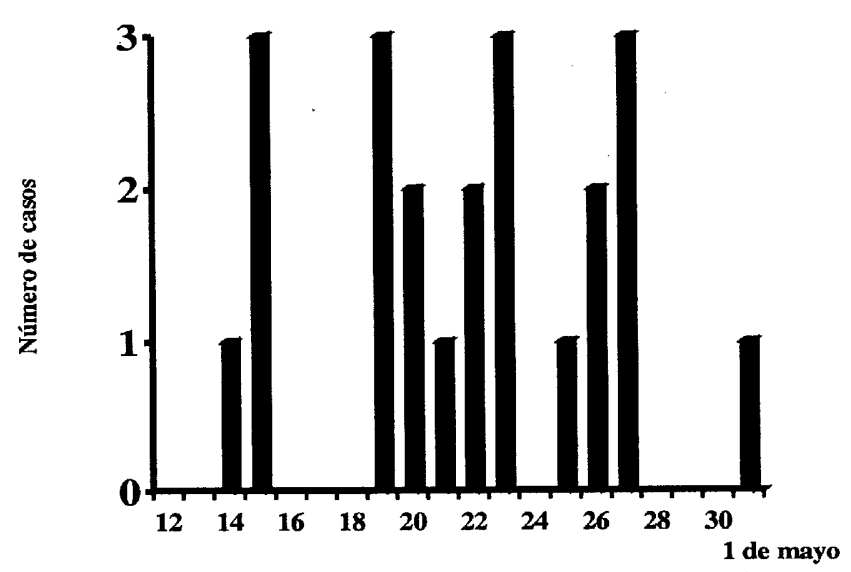


En el mismo municipio y por las mismas fechas se tuvo noticia también de un varón de 47 años, inmunodeficiente y hospitalizado, al que también se le visualizaron ooquistes de $C p$ en heces y que tuvo un cuadro compatible con la infección. Sin embargo, ya se encontraba en aquel momento con un cuadro de deterioro muy avanzado y falleció al poco tiempo por otras causas. Excepto en este caso, la evolución clínica fue satisfactoria, con medidas de rehidratación oral y tratamiento sintomático.

En cuatro de los casos confirmados se pudo realizar seguimiento de la excreción fecal de ooquistes. En todos se negativizó en menos de 2 meses, excepto en una niña de 3 años bien nutrida y sin patología previa que continuó eliminando $\mathrm{Cp}$ durante 6 meses.

\section{B) Estudio de casos y controles}

Veintiún casos y 24 controles fueron incluidos en el estudio de casos y controles. Al calcular las OR crudas para cada factor de riesgo investigado (tabla 2), se halló una relación estadísticamente significativa entre la enfermedad y la variable consumo de agua del grifo $(\mathrm{p}<0,05)$. También resultó significativa la variable tenencia de animales domésticos que aparece como factor protector $(\mathrm{OR}=0,2 ; \mathrm{p}<0,05)$.

Tabla 2

Odds ratio e intervalos de confianza de los factores de riesgo

\begin{tabular}{|c|c|c|c|c|}
\hline \multirow{2}{*}{ Factor de riesgo } & \multicolumn{2}{|c|}{ Expuestos } & \multirow{2}{*}{$O R$} & \multirow{2}{*}{$I C(95 \%)$} \\
\hline & $n$ & $T A$ & & \\
\hline Consumo de agua del grifo & 33 & 57,6 & 6,79 & $1,13-70,33$ \\
\hline Consumo de agua embotellada & 19 & 52,6 & 1,52 & $0,39-5,86$ \\
\hline Consumo de leche a granel & 3 & 66,7 & 2,42 & $0,12-148,93$ \\
\hline Contacto con animales domésticos & 14 & 21,4 & 0,20 & $0,03 \cdot 0,98$ \\
\hline Escolarización & 26 & 53,8 & 2,00 & $0,51-8,04$ \\
\hline Comedor escolar (1) & 10 & 50 & 0,78 & $0,12-5,01$ \\
\hline
\end{tabular}

TA: tasa de ataque (porcentaje de casos entre los expuestos).

OR: odds ratio.

IC: intervalo de confianza de la odds ratio (se han calculado los límites exactos).

(1): se han realizado los cálculos sobre la base de los 26 escolarizados.

Se procedió a realizar un nuevo análisis de las variables consumo de agua del grifo y tenencia de animales domésticos, estratificando por el resto de factores estudiados, sin que se modificasen los resultados anteriores.

Se investigó también la existencia de alguna relación entre los casos y la presencia 
de familiares y/o vecinos con diarrea, con el resultado de $\chi^{2}=10,96(\mathrm{p}<0,001)$.

\section{C) Inspección del abastecimiento de agua y análisis microbiológico}

Parte del agua del abastecimiento municipal procede de la confluencia de tres manantiales existentes en la sierra próxima y es canalizada mediante tuberías de hierro fundido a un depósito. El resto del agua en origen procede de captaciones de aguas superficiales. Este agua de doble procedencia, superficial y profunda, se mezcla en un depósito, en el que se somete a un tratamiento de desinfección mediante hipoclorito sódico y posteriormente se distribuye mediante tuberías de fibrocemento polietileno al la mayor parte del casco urbano.

Solamente dos zonas de poca población tenían un sistema de abastecimiento diferente, las denominadas Tablada y Cabezuelas. Esta última se abastece todo el año del agua gestionada por una empresa pública de ámbito autonómico.

Las captaciones de agua profunda no estaban valladas ni tenían delimitado un perímetro de protección. Durante la visita se observó ganado vacuno pastando en los alrededores. La recogida de agua superficial se hacía directamente del cauce mediante una canalización cuya única separación era una rejilla de hierro con unos barrotes de unos $5 \mathrm{~cm}$. de separación entre ellos; se observaron igualmente vacas pastando en las proximidades.

En los depósitos de almacenamiento se realizaba una decantación de los elementos de gran tamaño por la gravedad. No se disponía de otro sistema de tratamiento del agua previo a la desinfección.

Se tomaron muestras de agua en 4 zonas, en ninguna de las cuales se detectó la presencia de Cryptosporidium spp., si bien hay que señalar que en la muestra de agua superficial apareció gran cantidad de materia orgánica junto con algunas algas diatomeas.
En ninguno de los controles analíticos rutinarios, realizados durante el año 1997 y el 1998 hasta el inicio del brote, se detectaron alteraciones en los parámetros analizados, salvo niveles de cloro insuficientes o en exceso.

\section{D) Medidas de control}

Se remitió un informe del brote y sus posibles causas a las autoridades municipales que procedieron a realizar el cambio del sistema de abastecimiento de agua de la captación municipal por la de la empresa pública que explota los servicios de aducción y depuración de aguas en el ámbito autonómico y que ya se ha mencionado antes.

Durante los tres meses posteriores al brote se instauró un sistema de vigilancia especial para la detección de casos de diarrea que tuvieran una duración superior a 5 días, que incluyó a todos los médicos que atienden a personas residentes en el municipio. A lo largo de ese período no se tuvo noticia de ningún caso nuevo de diarrea con esas características.

\section{DISCUSIÓN}

Se puede afirmar que el brote por $C p$ estudiado estuvo centrado en Guadarrama. Aunque entre los casos hubo algunos niños que no eran residentes en el término municipal, todos, sin embargo, tenían relación con este pueblo, al menos por acudir al colegio. Por otra parte, no ha habido notificación de cuadros similares en municipios cercanos. Esta última información se constató entre los médicos con ejercicio en la zona. No sólo fueron afectados los niños: se detectó $C p$ en las heces de un varón con síndrome de inmunodeficiencia humana adquirida, que inició un cuadro diarreico en las fechas en que se produjo el brote. También dos mujeres adultas, previamente sanas, acudieron a consulta en la misma época aquejando diarrea de larga duración. El no haber detectado más casos en 
adultos sin patología subyacente previa puede ser debido a que la población mas susceptible a la infección por $C p$ son los niños y adultos inmunodeprimidos ${ }^{21,24,31}$, afectando en menor grado a personas adultas sanas en los que el cuadro clínico suele ser mas leve. Según algún estudio, durante el crecimiento se produce la maduración de ciertos mecanismos inmunitarios que proporcionan defensas suficientes frente a este parásito ${ }^{32}$. Además, es sabido que los adultos solicitan con menor frecuencia atención médica por este tipo de problemas y suelen automedicarse ${ }^{33}$. En este sentido se consideró que sería más eficiente dirigir la investigación epidemiológica hacia los grupos de edad que parecía que habían sido más afectados.

Aunque en el estudio de casos y controles que se realizó sólo se han incluido $21 \mathrm{ca}$ sos confirmados o probables, sin embargo, la magnitud del brote fue mayor. De hecho, aparte de los adultos señalados más arriba, hubo cinco niños más que cumplieron los criterios de caso en las fechas en que tuvo lugar el brote pero de los que no se tuvo conocimiento hasta después de concluir el estudio de casos y controles. Por otra parte, para mejorar la especificidad de la definición de caso, se incluyeron sólo los niños que tuvieron una diarrea de al menos cinco días de duración. Se sabe que muchos niños faltaron a clase durante los días del brote pero en la mayor parte de los casos fueron cuadros más leves o de menor duración, que no solicitaron asistencia médica o en los que no se investigó la presencia de $C p$ en las heces. En cualquier caso, se detectó una asociación significativa con el consumo de agua del grifo. La causalidad de este factor está también sustentada por otros datos epidemiológicos: la aparición de muchos casos en un corto periodo de tiempo, la dispersión geográfica de los casos dentro del término municipal y la presencia de afectados en distintas guarderías infantiles y centros educativos así como la afectación de niños no escolarizados. Hay que añadir, por último, que no se detectaron más casos tras el cambio del agua de la captación propia municipal por la de otra empre- sa de ámbito autonómico, que se realizó como medida preventiva. Por otra parte, la fuerte asociación entre los casos y la presencia de diarrea en familiares y/o vecinos, sugiere un mecanismo de transmisión, probablemente simultáneo al del consumo de agua del grifo, del tipo persona a persona.

En cuanto a las otras posibles fuentes de infección estudiadas, no se halló relación entre la enfermedad y el consumo de leche no pasteurizada, la visita a granjas o el baño en aguas recreativas. Sin embargo se encontró que la tenencia de animales de compañía actuaba como factor protector frente a la enfermedad. Este resultado es similar al observado en el brote de Milwaukee ${ }^{25}$. En general, no se ha implicado a los animales de compañía como transmisores del parásito, aunque algún estudio los hace responsables (cuando son menores de 6 meses), de la transmisión a personas VIH positivas ${ }^{21}$. Se podría aventurar que, en las personas con el sistema inmune sano, la convivencia con mascotas les hace desarrollar inmunidad al $C p-0$ inmunidad cruzada a otras especies de Cryptosporidium no patógenas para cl hombrc-que evita que desarrollen la enfermedad.

La presencia de ganado vacuno en las proximidades de aguas superficiales que forman parte del caudal utilizado para el consumo público explicaría la contaminación de esas aguas con ooquistes de $C p$ frecuentemente detectados en este tipo de ganado ${ }^{11,16,21}$. Debido a que los procedimientos de tratamiento de aguas de la captación municipal eran completamente ineficaces en la eliminación de ooquistes, ya que ćstos no sc ven afectados por la cloración, es posible el paso de estos ooquistes a las aguas de consumo ${ }^{21}$. En realidad, hasta el momento no se dispone de ningún sistema de tratamiento de aguas que sea absolutamente eficaz para el control de este parásito. Sin embargo se puede lograr una importante disminución de su concentración mediante sistemas de filtración ${ }^{4,34}$. En el caso que nos ocupa el tratamiento del agua no incluía ningún sistema de filtración. El hecho de no aislar el microorganismo en las 
aguas de consumo publico, es explicable debido a la dificultad técnica que ello supone ya que, con los medios diagnósticos de uso habitual, se deben procesar en el laboratorio grandes cantidades de agua parar la detección del parásito. Así, sólo en una pequeña proporción de brotes atribuidos a $C p$ es posible el hallazgo del parásito en el agua 11,13,17. Con todo, según los criterios utilizados por los Centers of Disease Control, se podrían incluir los resultados de este estudio dentro de los que tienen suficientes pruebas como para poder considerar que el $C p$ fue el causante de un brote de gastroenteritis transmitida por vía hídrica ${ }^{34}$.

Aunque en la literatura médica se han comunicado cierto número de brotes de diarrea producida por $C p$ consideramos que en España este es uno de los primeros de los que se informa. Esto no significa que este parásito sea infrecuente en nuestro medio, ya que se han realizado estudios de laboratorio que demuestran la presencia del parásito en heces especialmente en niños ${ }^{35}$. Además el Sistema de Información Microbiológica de la Red Nacional de Vigilancia Epidemiológica recoge semanalmente las identificaciones de $C p$ que se producen en los laboratorios declarantes. Sin embargo, la no comunicación de brotes nos lleva a pensar que corresponden a casos esporádicos. Se ha informado que en Inglaterra y Gales el $C p$ es la cuarta causa en importancia de diarrea confirmada por laboratorio ${ }^{12}$ y en Estados Unidos se ha establecido una norma legal que apoya la búsqueda de este parásito en los análisis ordinarios de agua potable ${ }^{36}$. Debido a la importancia que parece tener la criptosporidiosis como causa de diarrea, los médicos y pediatras de atención primaria han de tener muy presente este proceso en el diagnóstico diferencial de las diarreas.

Creemos que son necesarios nucvos estudios que profundicen en la mejora de métodos de detección del parásito, tratamiento de las aguas y tratamiento de esta enfermedad ya que en personas inmunodeprimidas puede llegar a suponer un grave compromiso para la vida ${ }^{28}$.

\section{AGRADECIMIENTOS}

A las doctoras Rocío Martínez y Beatriz Orden, del Laboratorio de Microbiología del Centro de Especialidades Argiielles de Madrid (Insalud), por su colaboración en el estudio diagnóstico de parásitos.

\section{BIBLIOGRAFÍA}

1. Nime FA, Burek JD, Page DL, Holsher MA, Yardley JH. Acute enterocolitis in a human being infected with the protozoan Cryptosporidium. Gastroenterology 1976;70:592-98.

2. Guerrant R.L. Cryptosporidiosis: An emerging, highly infectious threat. Emerging Infect Dis 1997;3 (1):1-9.

3. Holmberg SD, Moorman AC, von Bargen JC, Palella FJ, Loveless MO, Ward DJ et al. Posible eficacia de claritromicina y rifabutina para la quimioprofilaxis de la criptosporidiosis en la infección por VIH. JAMA (Ed Esp) 1998;7 (7):278-81.

4. Juranek DD. Cryptosporidiosis: Sources of infection and guidelines for prevention. Clin Infect Dis 1995;21 (Suppl 1):S57-61.

5. Sallon S, Deckelbaum RJ, Schmid II, Harlap S, Baras M, Spira DT. Cryptosporidium, malnutrition, and chronic diarrhea in children. Am J Dis Child 1988;142:312-5.

6. Macfarlane DE, Horner-Bryce J. Cryptosporidiosis in wellnourished and malnourished children. Acta Paediatr Scand 1987;76:474-7.

7. Millard PS, Gensheimer KF, Addis DG, Sosin DM, Beckett GA, Houck-Jankoski A et al. An outbreak of cryptosporidiosis from fresh-pressed apple cider. JAMA 1994 Nov;272 (20): 1592-6.

8. Chen YG, Yao FB, Li HS, Shi WS, Dai MX, Lu M. Cryptosporidium infection and diarrea in rural and urban areas of Jiangsu, Pcoples Rcpublic of China. J Clin Microbiol 1992 Feb;30 (2):492-4.

9. McAnulty JM, Fleming DW, González AH. A community-wide outbreak of cryptosporidiosis associated with swimming at a wave pool. JAMA 1994 Nov;272 (20):1597-600. 
10. Heigbel H, Slaine K, Seigel B, Wall P, McNabb SJ, Gibbons $\mathrm{H}$ et al. Outbreak of diarrhea in a day care center with spread to household members: the role of Cryptosporidium. Pediatr Infect Dis J 1987 Jun;6 (6):532-5.

11. Richardson AJ, Frankenberg RA, Buck AC, Selkon JB, Colbourne JS, Parsons JW et al. An outbreak of waterborne cryptosporidiosis in Swindon and Oxfordshire. Epidemiol Infect 1991;107:485-95.

12. Joseph C, Hamilton G, OConnor M, Nicholas S, Marshall R, Stanwell-Smith R et al. Cryptosporidiosis in the isle of Thanet; an outbreak asociated with local drinking water. Epidemiol Infect 1991;107:509-19.

13. Joce RE, Bruce J, Kiely D, Noah ND, Dempster WB, Stalker R et al. An outbreak of cryptosporidiosis associated with a swimming pool. Epidemiol Infect 1991;107:497-508.

14. D'Antonio RG, Winn RE, Taylor JP, Gustafson TL, Curent WL, Rhodes MM et al. Waterborne outbreak of cryptosporidiosis in normal hosts. Ann Intern Med 1985;103 (6 pt 1): 886-8.

15. Moodley D, Jackson TF, Gathiram V, van den Ende J. Cryptosporidium infections in children in Durban. Seasonal variation, age distribution and disease status. S Afr Med J 1991 Mar;79 (6):295-7.

16. Miron D, Kenes J, Dagan R. Calves as a source of an outbreak of cryptosporidiosis among young children in an agricultural closed community. Pediatr Infect Dis J 1991 Jun;10 (6):438-41.

17. Smith HV, Patterson WJ, Hardie R, Greene LA, Benton C, Tulloch W et al. An outbreak of waterborne cryptosporidiosis caused by posttreatment contamination. Epidemiol Infect 1989;103:703-15.

18. CDC. Outbreak of cryptosporidiosis at a Day Camp, Florida, July-August 1995. MMWR 1996;45 (21):443-5.

19. Dupont HL, Chappel CL, Sterling CR, Okhuisen PC, Rose JB, Jakubowski W. The infectivity of Cryptosporidium parvum in healthy volunteers. N Engl J Med 1995 Mar;332 (13):855-9.

20. Gallaher MM, Herndon JL, Nims LJ, Sterling CR, Grabowski DJ, Hull HF. Cryptosporidiosis and surface water. AJPH 1989 Jan;79 (1):3942 .
21. Casemore DP. Epidemiological aspects of human cryptosporidiosis. Epidemiol Infect 1990; 104:1-28.

22. Soave R, Weikel CS. Cryptosporidium y otros protozoarios incluyendo Isospora, Sarcocystis, Balantidium coli y Blastocystis. En: Mandel GL, Gordon Douglas R, Bennett JE (directores). Enfermedades infecciosas. Principios y práctica. $3^{a}$ ed. Buenos Aires: Editorial Médica Panamericana; 1992.p.2253-61.

23. Mac Kenzie WR, Schell WL, Blair KA, Addis DG, Peterson DE, Hoxie NI et al. Massive outbreak of waterborne Cryptosporidium infection in Milwaukee, Wisconsin: Recurrence of ilness and risk of secundary transmission. Clin Infect Dis 1995 Jul;21:57-62.

24. Goldstein ST, Juranek DD, Ravenholt O, Hightower AW, Martin DG, Mesnik JL et al. Cryptosporidiosis: an outbreak associated with drinking water despite state-of-the-art watcr treatment. Ann Intern Med 1996 Mar; 124 (5):459-68.

25. Mac Kenzie WR, Hoxie NJ, Proctor ME, Gradus MS, Blair KA, Peterson DE et al. A massive outbreak in Milwaukee of criptosporidium infection transmitted trough the public water supply. N Engl J Med 1994 Jul;331:161-7.

26. Petry F. Epidemiological study of Cryptosporidium parvum in sera of persons from Gcrmany. Infection 1998;26 (1):1-11.

27. Harp JA, Fayed R, Pesch BA, Jackson GJ. Effect of pasteurization on infectivity of Cryptosporidium parvum. Oocysts in Water and Milk. Appl Environ Microbiol 1996 Aug;62 (8): 2866-8.

28. Herwaldt BL, Craun GF, Stokes SL, Junarek DD. Waterborne-Disease Outbreaks, 1989 1990. MMWR 1991;40 (SS-3):1-21.

29. Proctor ME, Blair KA, Davis JP. Surveillance data for waterborne illness detection: an assesment following a massive waterborne outbreak of Cryptosporidium infection. Epidemiol Infect 1998;120:43-5.

30. Henrikson SA, Pohlenz JFL. Staining of cryptosporidia by a modified Ziehl-Neelsen technique. Acta Vet Scand 1981;22:594-6.

31. Perz JF, Ennever FK, Le Blancq SM. Cryptosporidium in tap water: comparison of predicted risk with observed levels of disease. Am J Epidemiol 1998:47 (3):289-301. 
32. Theodos CM. Innate and Cell-mediated Immune Responses to Cryptosporidium parvum. Adv Parasitol 1998;40:87-119.

33. Nebot M, Llauger MA. Autocuidado de los trastornos comunes de salud: resultados de una encuesta telfónica en la población general. Med Clin (Barc) 1992 Oct 10;99 (11):420-4.

34. CDC. Surveillance for Waterborne-Disease Outbreaks-United States, 1993-1994. MMWR 1996;45 (SS-1):1-33.
35. López-Brea M, García Picazo L, Del Rey MC. Cryptosporidium in children and adults in Madrid (carta). J Infect 1986 Nov; 13 (3):3145.

36. United States Environmental Protection Agency. Office of Water. Information Collection Rule. Optional Public Notice Language for Cryptosporidium. EPA 811-F-96-007. Federal Register Notice, May 14, 1996. URL: http://www. epa.gov/OGWDW/icr_not.html. 